\title{
Confirmation of persistent radio jets in the microquasar LS 5039
}

\author{
J. M. Paredes ${ }^{1}$, M. Ribó ${ }^{1}$, E. Ros $^{2}$, J. Martî $^{3}$, and M. Massi ${ }^{2}$ \\ ${ }^{1}$ Departament d'Astronomia i Meteorologia, Universitat de Barcelona, Av. Diagonal 647, 08028 Barcelona, Spain \\ 2 Max-Planck-Institut für Radioastronomie, Auf dem Hügel 69, 53121 Bonn, Germany \\ 3 Departamento de Física, Escuela Politécnica Superior, Universidad de Jaén, Virgen de la Cabeza 2, 23071 Jaén, Spain
}

Received 5 August 2002 / Accepted 27 August 2002

\begin{abstract}
We present here new observations conducted with the EVN and MERLIN of the persistent microquasar LS 5039 discovered by Paredes et al. (2000) with the VLBA. The new observations confirm the presence of an asymmetric two-sided jet reaching up to $\sim 1000 \mathrm{AU}$ on the longest jet arm. The results suggest a bending of the jets with increasing distance from the core and/or precession. The origin and location of the high-energy gamma-ray emission associated with the system is discussed and an estimate of the magnetic field at the base of the jet given. Our results suggest a well collimated radio jet. We also comment on new observing strategies to be used with satellites and forthcoming detectors, since this persistent source appears to be a rather good laboratory to explore the accretion/ejection processes taking place near compact objects.
\end{abstract}

Key words. stars: individual: LS 5039, RX J1826.2-1450, 3EG J1824-1514 - X-rays: binaries - radio continuum: stars radiation mechanism: non-thermal

\section{Introduction}

Microquasars are radio emitting X-ray binaries with relativistic jets, like SS 433, GRS 1915+105, GRO J1655-40 or Cygnus X-3. The reader is referred to Mirabel \& Rodríguez (1999) for a detailed review and to Castro-Tirado et al. (2001) and Durouchoux et al. (2002) for the most recent studies of microquasars.

The $V=11.2$ star LS 5039, located at an estimated distance of $\sim 3 \mathrm{kpc}$ and close to the galactic plane $\left(l=16.88^{\circ}\right.$, $b=-1.29^{\circ}$ ), was initially proposed by Motch et al. (1997) to be the optical counterpart of the X-ray source RX J1826.2-1450, a likely High Mass X-ray Binary (HMXB). Using data from the NRAO VLA Sky Survey and follow-up VLA observations, Martí et al. (1998) discovered that the source was also a non-thermal radio emitter with moderate variability. X-ray observations of RX J1826.2-1450 carried out by Ribó et al. (1999) showed that the X-ray spectrum was significantly hard up to $30 \mathrm{keV}$, with a strong Gaussian iron line at $6.6 \mathrm{keV}$, and neither pulsed nor periodic emission was found on time scales of $0.02-2000 \mathrm{~s}$ and 2-200 days, respectively. Paredes et al. (2000) discovered that the system displays relativistic radio jets at milliarcsecond (mas) scales, revealing the microquasar nature of LS 5039, and proposed an association with the $\gamma$-ray source 3EG J1824-1514. While in the past the mass donor had been classified as an $\mathrm{O} 7 \mathrm{~V}((\mathrm{f}))$ star, optical and near-IR spectroscopic observations by Clark et al. (2001) show that it is in fact

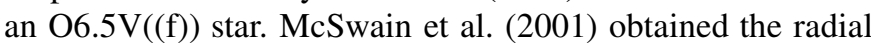

Send offprint requests to: J. M. Paredes,

e-mail: josep@am.ub.es velocity curve of the system, with their fitted parameters being a short period of $P=4.117$ days, a high eccentricity of $e=0.41$, a radial velocity of the system of $V_{0}=4.6 \mathrm{~km} \mathrm{~s}^{-1}$ and a mass function of $f(m)=0.00103 M_{\odot}$. Ribó et al. (2002) obtained optical and radio proper motions, and found that LS 5039 is a runaway X-ray binary with a space velocity $\sim 150 \mathrm{~km} \mathrm{~s}^{-1}$. McSwain \& Gies (2002), based on wind accretion models, have recently suggested a neutron star as the compact object in LS 5039, and proposed an inclination of $i \simeq 30^{\circ}$ for this binary system.

The persistent radio emission of LS 5039 suggests that the jet is always present. With the aim to confirm this hypothesis, and also to detect the jet at larger angular scales than those imaged with the previous Very Long Baseline Array (VLBA) measurements, we observed this microquasar with the European VLBI Network (EVN) and the Multi-Element RadioLinked Interferometer Network (MERLIN). In this Letter we present the obtained results.

\section{Observations and results}

We observed LS 5039 simultaneously with the EVN and MERLIN on March 12000 (3:20-7:10 UT, MJD 51604.2) at $5 \mathrm{GHz}$. The EVN observations were performed with 7 antennas, namely (antenna, location, diameter) Effelsberg, Germany, 100 m; Jodrell Bank, UK, 25 m; Cambridge, UK, $32 \mathrm{~m}$; Westerbork, The Netherlands, $14 \times 25 \mathrm{~m}$; Medicina, Italy, $32 \mathrm{~m}$; Noto, Italy, $32 \mathrm{~m}$; and Toruń, Poland, $32 \mathrm{~m}$. Data were recorded in MkIV mode with 2-bit sampling at $256 \mathrm{Mbps}$ at left hand circular polarization, yielding a full bandwidth 


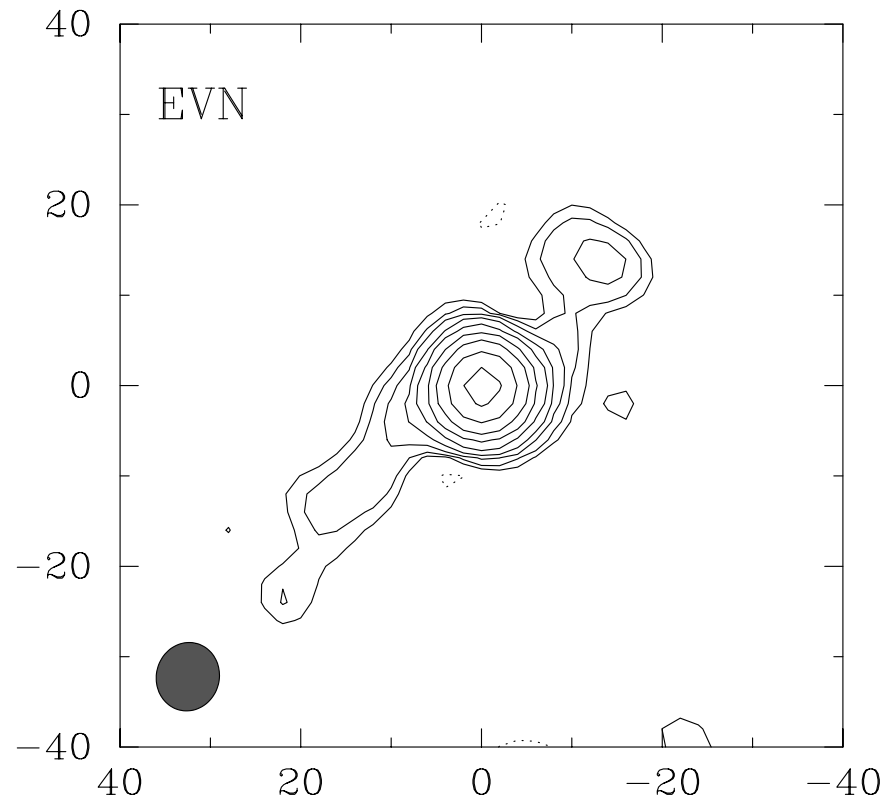

Fig. 1. EVN self-calibrated image of LS 5039 at $5 \mathrm{GHz}$ obtained on March 1 2000. Axes units are in mas. The synthesized beam, plotted in the lower left corner, has a size of $7.60 \times 6.96$ mas in PA of $-14^{\circ}$. The first contour corresponds to $0.3 \mathrm{mJy} \mathrm{beam}^{-1}$, while consecutive ones scale with $3^{1 / 2}$.

of $64 \mathrm{MHz}$. The data were correlated at the Joint Institute for VLBI in Europe (JIVE). Standard fringe fitting and imaging analysis were carried out using AIPS and DIFMAP. MERLIN recorded data with 2-bit sampling at dual polarization and a total $32 \mathrm{MHz}$ bandwidth. We analyzed the left hand circular polarization data excluding $1 \mathrm{MHz}$ channel at both edges of the band, yielding a final bandwidth of $14 \mathrm{MHz}$.

Inspection of the shortest MERLIN baselines reveals a constant flux density during the full run. That allowed us to image the source directly without splitting the data in time blocks. We present the obtained EVN and MERLIN images in Figs. 1 and 2, respectively. The MERLIN image is presented convolved with a circular beam, equivalent in solid angle to the interferometric synthesized beam of $142 \times 46$ mas at position angle (PA) $-47^{\circ}$. Our images clearly show that LS 5039 has a bipolar jet emanating from a central core. In both images the southeast (SE) jet is brighter and larger than the northwest (NW) one, as can be seen in Table 1, where we have quoted the flux densities of the core and the jets and the lengths and PA of the jets. Their total lengths are $\sim 60$ mas in the EVN image and $\sim 300$ mas in the MERLIN one.

\section{Jet parameters and intrinsic sizes}

These results confirm the existence of a two-sided radio jet in LS 5039 reported in previous VLBA $5 \mathrm{GHz}$ observations by Paredes et al. (2000). This source does not present any strong outburst or, at least, none has been detected during the eleven month monitoring carried out by the Green Bank Interferometer between 1998 September 16 and 1999 August 22 (Clark et al. 2001). On the other hand, inspection of the RXTE All Sky Monitor data (Levine et al. 1996) reveals

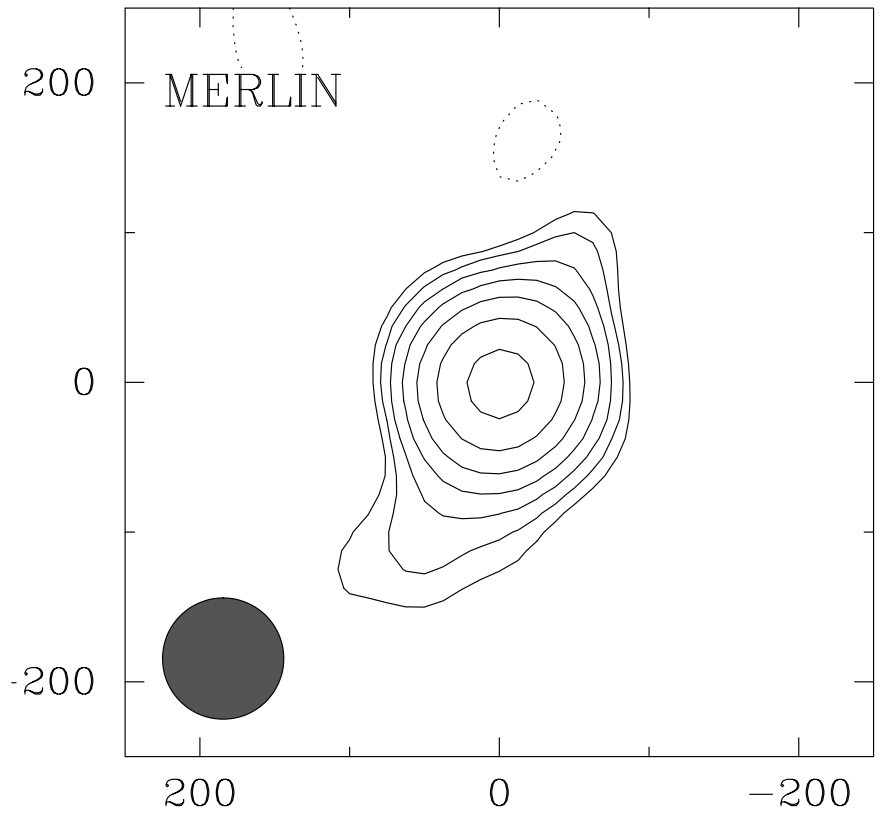

Fig. 2. MERLIN self-calibrated image of LS 5039 at $5 \mathrm{GHz}$ obtained on March 1 2000. Axes units are in mas. The convolving circular beam, plotted in the lower left corner, has a diameter of 81 mas. The first contour corresponds to a flux density of $1 \mathrm{mJy}^{\text {beam }}{ }^{-1}$, while consecutive ones scale with $3^{1 / 2}$.

Table 1. Flux densities at $5 \mathrm{GHz}$, length and PA of the LS 5039 structure features as detected by EVN and MERLIN.

\begin{tabular}{lrccccc}
\hline \hline & \multicolumn{3}{c}{ EVN } & \multicolumn{3}{c}{ MERLIN } \\
\hline & $\begin{array}{l}S_{5 \mathrm{GHz}} \\
{[\mathrm{mJy}]}\end{array}$ & $\begin{array}{c}\text { Length } \\
{[\mathrm{mas}]}\end{array}$ & $\begin{array}{c}\text { PA } \\
{\left[{ }^{\circ}\right]}\end{array}$ & $\begin{array}{c}S_{5 \mathrm{GHz}} \\
{[\mathrm{mJy}]}\end{array}$ & $\begin{array}{c}\text { Length } \\
{[\mathrm{mas}]}\end{array}$ & $\begin{array}{c}\text { PA } \\
{\left[{ }^{\circ}\right]}\end{array}$ \\
\hline Core & 29.3 & - & - & 31.6 & - & - \\
NW jet & 2.6 & 24 & -42 & 4.0 & 128 & -29 \\
SE jet & 3.3 & 34 & 140 & 4.2 & 174 & 150 \\
\hline
\end{tabular}

Note: the errors in flux density, lenght and PA are $0.1 \mathrm{mJy}^{-}$beam ${ }^{-1}$, 2 mas and $4^{\circ}$ for the EVN features and $0.4 \mathrm{mJy}_{\text {beam }}{ }^{-1}, 12$ mas and $5^{\circ}$ for the MERLIN ones.

that the X-ray flux was at the typical low level (Ribó et al. 1999) at the epoch of the EVN and MERLIN observations and several weeks before. All this suggests that the jets are persistent, as the VLBI images obtained up to now seem to indicate.

In the observations reported here, the jets extend further away than the $\sim 6$ mas of the VLBA observations at the same frequency. In all the images the jets have similar position angles, $\sim 125^{\circ}$ in the VLBA image from Paredes et al. (2000), $\sim 140^{\circ}$ in our EVN image, and $\sim 150^{\circ}$ in our MERLIN image. These results suggest a bending of the jets with increasing distance from the core and/or precession.

The brightness and length asymmetry of the jet components may involve special relativity effects (Mirabel \& Rodríguez 1999). Hence, assuming that this is the reason for the detected 
length asymmetry of the jets, we can estimate some parameters by using the following equation:

$\beta \cos \theta=\frac{\mu_{\mathrm{a}}-\mu_{\mathrm{r}}}{\mu_{\mathrm{a}}+\mu_{\mathrm{r}}}=\frac{d_{\mathrm{a}}-d_{\mathrm{r}}}{d_{\mathrm{a}}+d_{\mathrm{r}}}$,

where $\beta$ is the velocity of the jet flow in units of the speed of light, $\theta$ is the angle between the direction of motion of the ejecta and the line of sight and $\mu_{\mathrm{a}}$ and $\mu_{\mathrm{r}}$ are the proper motions of the approaching (SE) and receding (NW) components, respectively. Although we do not know the epoch of ejection of the terminal plasma, we can cancel the time variable by using the relative distances to the core $d_{\mathrm{a}}$ and $d_{\mathrm{r}}$, as expressed in (1). We will identify $d_{\mathrm{a}}$ and $d_{\mathrm{r}}$ with the lengths of the approaching and receding jet, respectively. Using (1) and the EVN values in Table 1 we obtain $\beta \cos \theta=0.17 \pm 0.05$, and hence $\beta>0.17 \pm 0.05$ and $\theta<80^{\circ} \pm 3^{\circ}$. For the MERLIN values we obtain $\beta>0.15 \pm 0.06$ and $\theta<81^{\circ} \pm 3^{\circ}$. These values are similar to those previously derived from the VLBA image, of $\beta>0.15 \pm 0.04$ and $\theta<81^{\circ} \pm 2^{\circ}$. We have not considered an eventual larger size for the NW jet with a brightness below the image noise level. McSwain \& Gies (2002) have recently proposed an inclination of $i \simeq 30^{\circ}$ for LS 5039. If we assume that the jet is perpendicular to the accretion disk, and that the disk lies in the orbital plane of the binary system, then $\theta=i=30^{\circ}$, and using the values from the EVN image, we obtain $\beta=0.20 \pm 0.06$, which indicates a mildly relativistic jet.

The total length of the EVN and MERLIN jets is $\sim 60$ and $\sim 300$ mas, respectively. Considering that the source is located at $2.9 \mathrm{kpc}$ (Ribó et al. 2002), these angular lengths translate into linear lengths in the plane of the sky of $\sim 175$ and $\sim 870 \mathrm{AU}$, respectively. Assuming that $\theta=30^{\circ}$ we obtain intrinsic total lengths of $\sim 350$ and $\sim 1740$ AU, respectively, and lengths of the SE jet arm of $\sim 200$ and $\sim 1000$ AU, respectively. Moreover, the jet width is smaller than one synthesized beam even in the EVN image. This implies a jet half opening angle $\leq 6^{\circ}$.

\section{A scenario based on the $\gamma$-ray/radio emission}

It is interesting to use the presence of $5 \mathrm{GHz}$ synchrotron emitting particles so far away in order to constrain the jet physical parameters. The starting point of the following calculations will be the scenario for the proposed EGRET emission of LS 5039 presented in Paredes et al. (2000). In this context, the high energy $\gamma$-ray photons arise from inverse Compton (IC) scattering of UV photons from the luminous companion star by the relativistic electrons that later, after having lost part of their original energy due to IC losses, will account for the radio emission in the jets.

We will adopt here a very simple model of an expanding jet. Cylindrical coordinates, $z$ and $r$ measured parallel and perpendicular to the jet axis, are the best choice in our case. The jet is assumed to form at a distance $z_{0}$ from the compact object and flows with a velocity $v=\beta c$. The lateral expansion of the jet is parametrized as $r=r_{0}\left(z / z_{0}\right)^{\epsilon}$, where $r_{0}$ is the initial jet radius. A freely expanding conical jet would correspond to $\epsilon=1$, while for slowed lateral expansion we would have $\epsilon<1$ (see e.g. Hjellming \& Johnston 1988). The expansion velocity perpendicular to the jet axis is thus $v_{\perp}=\mathrm{d} r / \mathrm{d} t=\epsilon v r / z$. Concerning the magnetic field, we will only consider the component perpendicular to the jet axis, because it has the slowest decay if conservation of the magnetic flux is assumed. The magnetic field along the jet will be thus parametrized as $B=B_{0}\left(r / r_{0}\right)^{-1}=B_{0}\left(z / z_{0}\right)^{-\epsilon}$.

A relativistic electron injected at the base of the jet will decrease its energy mainly through adiabatic expansion, IC and synchrotron losses according to:

$\frac{\mathrm{d} E}{\mathrm{~d} t}=-\frac{2}{3} \frac{v_{\perp} E}{r}-\alpha_{\mathrm{IC}} U_{\mathrm{rad}} E^{2}-\alpha_{\mathrm{S}} B^{2} E^{2}$.

The factor $2 / 3$ in the adiabatic expansion term comes from the lateral expansion of the jet. The constants $\alpha_{\mathrm{IC}}=3.97 \times 10^{-2}$ and $\alpha_{\mathrm{S}}=2.37 \times 10^{-3}$ (cgs units) are the coefficients of the terms accounting for IC and synchrotron losses, respectively. The radiation energy density $U_{\text {rad }}$ is assumed to be dominated by stellar UV photons from the O6.5V star, whose luminosity above $10 \mathrm{eV}$ amounts to $L_{*} \simeq 5 \times 10^{38} \mathrm{erg} \mathrm{s}^{-1}$ and $U_{\text {rad }}=L_{*} /\left[4 \pi c\left(a^{2}+z^{2}\right)\right]$. With the recently determined orbital parameters (McSwain et al. 2001; McSwain \& Gies 2002), the likely semimajor axis of the orbit is $a=2.6 \times 10^{12} \mathrm{~cm}$. Adopting these parameters, the radiation energy density close to the compact object is so high that electrons with energies higher than $10^{-3}$ erg will easily produce $\gamma$-ray photons with energies of 100-1000 MeV, as detected by EGRET. Moreover, integration of (2) taking only into account the IC losses, reveals that the energy of the electrons decays very fast to values of $\sim 5 \times 10^{-4} \mathrm{erg}$, regardless of their initial energy.

The IC contribution in (2) should be comparable to adiabatic losses at least at the base of the jet. Later on, the adiabatic expansion term will soon become dominant due to its slower decay $\left(\propto z^{-1}\right)$. Therefore, at the base of the jet we can request:

$\alpha_{\mathrm{IC}} \frac{L_{*}}{4 \pi c\left(a^{2}+z_{0}^{2}\right)} E_{0}^{2} \simeq \frac{2 \epsilon}{3} \frac{v E_{0}}{z_{0}}$.

We can use the values quoted above, together with $v=0.2 c$ and an energy of $E_{0}=5 \times 10^{-4} \mathrm{erg}$ for the electrons injected at the base of the jet, to solve for $z_{0}$ in this quadratic equation. The obtained results are $0.35,0.85$ and 1.30 AU for $\epsilon=1,1 / 2$ and $1 / 3$, respectively. The synchrotron term can be shown to be negligible a posteriori and has not been considered here.

Hereafter, we will proceed taking into account only the adiabatic expansion term, because it is dominant compared to the IC one, and rewrite (2) as:

$\frac{\mathrm{d} E}{\mathrm{~d} t} \simeq-\frac{2 \epsilon}{3} \frac{v E}{z}$.

This can be easily integrated to give $E \simeq E_{0}\left(z / z_{0}\right)^{-2 \epsilon / 3}$. As the energy decays, the synchrotron emission of relativistic electrons will shift towards lower frequencies. In particular, the synchrotron frequency expected at a distance $z$ is given by:

$v_{\mathrm{c}}=6.27 \times 10^{18} B E^{2} \simeq 6.27 \times 10^{18} B_{0} E_{0}^{2}\left(z / z_{0}\right)^{-7 \epsilon / 3}$.

The electrons still producing $6 \mathrm{~cm}\left(v_{\mathrm{c}}=5 \times 10^{9} \mathrm{~Hz}\right)$ synchrotron emission at about $z=1000$ AU (the size of the 
MERLIN jets) are probably those originally injected with energies of $E_{0} \simeq 5 \times 10^{-4} \mathrm{erg}$. Then, the magnetic field $B_{0}$ at the base of the jet can be roughly estimated as:

$B_{0} \simeq 8.0 \times 10^{-10} E_{0}^{-2}\left[\frac{z}{z_{0}}\right]^{7 \epsilon / 3}$.

This provides values of $3.6 \times 10^{5}, 12$ and $0.6 \mathrm{G}$ for $\epsilon=1$, $1 / 2$ and $1 / 3$, respectively. If magnetic flux is conserved, the corresponding field at the end of the MERLIN jet would be $B_{1000 \mathrm{AU}}=128,0.4$ and $0.06 \mathrm{G}$. For comparison, the only independent estimates of the magnetic field in the LS 5039 jets come from simple equipartition arguments. The results are merely indicative of the average magnetic field in the solid angle of the sky covered by the jets. Using the total flux density and size of the MERLIN jets given in Table 1 we derive a field value of $>8 \times 10^{-3} \mathrm{G}$, although this is only a lower limit because the jet width is unresolved. The VLBA image by Paredes et al. (2000), allowed them to estimate an approximate magnetic field of $\sim 0.2 \mathrm{G}$ for radio jets reaching up to a few mas. It is clear that a field of such intensity is more consistent with the $\epsilon<1$ results than with the too high magnetic fields implied by a simple conical jet $(\epsilon=1)$. Therefore, a slowly expanding jet, and hence well collimated, is favoured to power the LS 5039 radio emission up to $\sim 1000 \mathrm{AU}$ by the electrons that previously contributed to the $\gamma$-ray emission, without in situ acceleration being required. This is also in agreement with our upper limit of $\leq 6^{\circ}$ for the jet half opening angle.

\section{Future prospects}

The high eccentricity of LS 5039 provides both, a variable accretion rate along the orbit implying a variable rate of electrons injected into the jet, and a variable radiation energy density close to the compact object. Hence, it is predicted a variability in the $\gamma$-ray luminosity correlated with the orbital period, which may hopefully be detected by INTEGRAL and/or GLAST. On the other hand, if the star exhibits intrinsic variations in the UV photon flux, a variability in $\gamma$-rays would be seen correlated to it.

Since LS 5039 seems to have persistent radio jets, it would be interesting to study the possibility of detecting Dopplershifted lines. As the jet flow has an estimated velocity of $\sim 0.2 c$, the amount of Doppler-shift is expected to be comparable to that found in SS 433 (Kotani et al. 1996; Marshall et al. 2002). Hence, spectroscopic X-ray observations with XMM or Chandra could be very useful to constrain the physics of the relativistic flow and to study the matter content within the jet. Finally, as pointed out by Levinson \& Waxman (2001) and by Distefano et al. (2002), if the jets are hadronic we would expect the formation of $\mathrm{TeV}$ neutrinos, that could be detected in the future with detectors of $\mathrm{km}^{2}$-scale effective area.

Overall, these results confirm the existence of persistent relativistic radio jets in LS 5039, indicating that this source is a very good target to be studied with new instruments in order to gain knowledge on jet physics.
Acknowledgements. We acknowledge J. García-Sánchez and A. Lobanov for their useful comments and suggestions after reading through a draft version of this paper. We acknowledge detailed and useful comments from L. F. Rodríguez, the referee of this Letter. We are very grateful to S. T. Garrington, M. A. Garrett, D. C. Gabuzda, and C. Reynolds for their valuable help in the data reduction process. This paper is based on observations with the $100-\mathrm{m}$ telescope of the MPIfR (Max-Planck-Institut für Radioastronomie) at Effelsberg. We thank the staff of the JIVE correlator and of the observing telescopes. The European VLBI Network is a joint facility of European, Chinese and other radio astronomy institutes funded by their national research councils. MERLIN is operated as a National Facility by the University of Manchester at Jodrell Bank Observatory on behalf of the UK Particle Physics \& Astronomy Research Council. The EVN observations were carried out thanks to the TMR Access to Large-scale Facilities programme under contract No. ERBFMGECT950012. Part of the data reduction was done at JIVE with the support of the European Community - Access to Research Infrastructure action of the Improving Human Potential Programme, under contract No. HPRI-CT-1999-00045. J.M.P., M.R. and J.M. acknowledge partial support by DGI of the Ministerio de Ciencia y Tecnología (Spain) under grant AYA2001-3092, as well as partial support by the European Regional Development Fund (ERDF/FEDER). During this work, M.R. has been supported by two fellowships from CIRIT (Generalitat de Catalunya, ref. 1998 BEAI 200293 and 1999 FI 00199). J.M. has been aided in this work by an Henri Chrétien International Research Grant administered by the AAS, and has been partially supported by the Junta de Andalucía.

\section{References}

Castro-Tirado, A. J., Greiner, J., \& Paredes, J. M. 2001, Microquasars, Proc. of the Third Microquasar Workshop, Ap\&SS, 276

Clark, J. S., Reig, P., Goodwin, S. P., et al. 2001, A\&A, 376, 476

Distefano, C., Guetta, D., Waxman, E., \& Levinson, A. 2002, ApJ, 575, 378

Durouchoux, Ph., Fuchs, Y., \& Rodriguez, J. 2002, Proc. of the Fourth Microquasar Workshop, published by the Center for Space Physics, Kolkata, India

Hjellming, R. M., \& Johnston, K. J. 1988, ApJ, 328, 600

Kotani, T., Kawai, N., Matsuoka, M., \& Brinkmann, W. 1996, PASJ, 48,619

Levine, A. M., Bradt, H., Cui, W., et al. 1996, ApJ, 469, L33

Levinson, A., \& Waxman, E. 2001, Phys. Rev. Lett., 87, 171101

Marshall, H. L., Canizares, C. R., \& Schulz, N. S. 2002, ApJ, 564, 941

Martí, J., Paredes, J. M., \& Ribó, M. 1998, A\&A, 338, L71

McSwain, M. V., \& Gies, D. R. 2002, ApJ, 568, L27

McSwain, M. V., Gies, D. R., Riddle, R. L., Wang, Z., \& Wingert, D. W. 2001, ApJ, 558, L43

Mirabel, I. F., \& Rodríguez, L. F. 1999, ARA\&A, 37, 409

Motch, C., Haberl, F., Dennerl, K., Pakull, M., \& Janot-Pacheco, E. 1997, A\&A, 323, 853

Paredes, J. M., Martí, J., Ribó, M., \& Massi, M. 2000, Science, 288, 2340

Ribó, M., Reig, P., Martí, J., \& Paredes, J. M. 1999, A\&A, 347, 518

Ribó, M., Paredes, J. M., Romero, G. E., et al. 2002, A\&A, 384, 954 